\title{
Association between basal stem rot disease and simple sequence repeat markers in oil palm, Elaeis guineensis Jacq.
}

\begin{abstract}
The oil palm is badly affected by basal stem rot (BSR) disease in Southeast Asia. BSR disease is caused by the fungus Ganoderma boninense, which is a major threat to oil palm compared with other Ganoderma spp. Molecular markers associated with BSR disease will accelerate the identification process of resistant breeding materials in screening of plants for tolerance to the disease at the nursery stage. In this study, 58 simple sequence repeat markers were utilized with three progeny types, namely, KA4G1, KA4G8, and KA14G8, to perform a comparative molecular mapping for association with BSR. A total of 319 alleles were identified with an average of 5.51 alleles per locus. Five markers, mEgCIR0793:180, mEgCIR0894:200, mEgCIR03295:210, mEgCIR3737:146 and mEgCIR3785:299 were found to be associated with Ganoderma disease with $\mathrm{P}$ values of $0.018,0.033,0.037,0.034$ and 0.037, respectively, in single progeny analysis. However, in pooled data (KA4G1, KA4G8 and KA14G8), only two alleles, mEgCIR0804:213 (P value $=0.001)$ and mEgCIR3292:183 $(\mathrm{P}$ value $=0.001)$, were found to be associated with Ganoderma disease. These analyses confirmed that progeny type KA4G1 was tolerant, whereas the other two were susceptible progeny types. These markers and KA4 progeny will be useful in future works on BSR disease resistance in oil palm.
\end{abstract}

Keyword: Association; Breeding for resistance; Ganoderma boninense; Oil palm; Simple sequence repeats 